Ronny Jahn

Der blinde Fleck im Spitzensport 
Soziologische Studien

Band 33 
Ronny Jahn

\section{Der blinde Fleck im Spitzensport}

\section{Zur soziologischen Begründung der Supervision und ihrer Anwendung im Leistungssport}

Centaurus Verlag \& Media UG 2008 


\section{Zum Autor:}

Ronny Jahn, geb. 1978, MA, studierte Soziologie, Politik und Medienwissenschaften und war Mitglied im Nachwuchsteam der Radsportprofimannschaft Team Deutsche Telekom. Er promoviert derzeit an der Universität Potsdam am Institut für Erziehungswissenschaft und ist als freiberuflicher Berater tätig.

\section{Die Deutsche Bibliothek - CIP-Einheitsaufnahme}

Bibliographische Information der Deutschen Bibliothek:

Die deutsche Bibliothek verzeichnet diese Publikation in der Deutschen Nationalbibliographie; detaillierte bibliographische Daten sind im Internet über http://dnb.ddb.de abrufbar.

ISBN 978-3-8255-0691-9

ISBN 978-3-86226-332-5 (eBook)

DOI 10.1007/978-3-86226-332-5

\section{ISSN 0937-664X}

Alle Rechte, insbesondere das Recht der Vervielfältigung und Verbreitung sowie der Übersetzung, vorbehalten. Kein Teil des Werkes darf in irgendeiner Form (durch Fotokopie, Mikrofilm oder ein anderes Verfahren) ohne schriftliche Genehmigung des Verlages reproduziert oder unter Verwendung elektronischer Systeme verarbeitet, vervielfältigt oder verbreitet werden.

(C) CENTAURUS Verlags KG, Kenzingen 2008

Umschlaggestaltung: Alexander Labrentz 


\section{Inhalt}

Vorwort

Einleitung

\section{Teil I: Theorie}

$\begin{array}{lr}\text { 1. Supervision } & 9\end{array}$

$\begin{array}{lll}1.1 & \text { Der Begriff Supervision } & 10\end{array}$

$\begin{array}{lll}1.2 & \text { Ursprünge aktueller Supervisionspraxis } & 11\end{array}$

1.3 Entwicklungsverläufe: Theorie und Praxis im Feld der Supervision 13

1.4 Die Ausbreitung supervisorischer Praxis in Deutschland 15

$\begin{array}{lll}1.5 & \text { Verlaufsskizze einer Supervision } & 17\end{array}$

1.6 Ausblick: Supervision im Kontext der Arbeit 19

$\begin{array}{ll}\text { 2. Die Theorie des kommunikativen Handelns } & 20\end{array}$

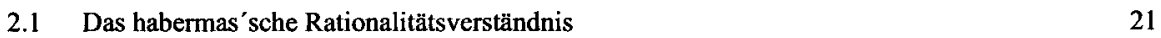

2.2 Die habermas'sche Handlungstypologie -
kommunikatives versus zweckrationales Handeln

2.3 Exkurs: Die Verbindung von Handlungs- und Systemtheorie - Lebenswelt und System 25

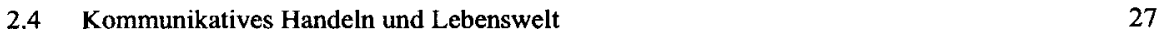

$\begin{array}{lll}2.5 & \text { Strategisches Handeln } & 36\end{array}$

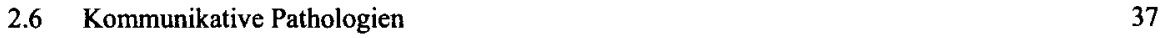

2.7 Ausblick: Die Theorie des kommunikativen Handelns im Kontext der Arbeit 40

3. Versuch einer Theoriesynthese -

Supervision: Die praktische Fortsetzung der Theorie kommunikativen Handelns 41

3.1 These (1): Die habermas'schen Geltungsansprüche und ihre Analogie zu den Tätigkeitsfeldern supervisorischer Praxis

3.2 These (2): Die Vision der idealen Sprechsituation Supervision als Medium zur Diskursfindung und -unterstïtzung $\quad 46$

3.3 Ausblick: Die Theoriesynthese im Kontext der Arbeit $\quad 51$ 


\section{Teil II: Qualitative Untersuchung}

\section{Rekonstruktion, ,sozialer Wirklichkeit" - „empirische Wirklichkeit“ theoretisch reflektiert}

4.1 Theoretische Anmerkungen zur angewandeten Methodologic: Verstehen durch sozialwissenschaftliche Hermeneutik

4.2 Methodische Umsetzung und Interviewauswahl $\quad 59$

$\begin{array}{lll}4.3 & \text { Feldeinführung und Feldzugang } & 62\end{array}$

4.4 Team Blau - Selbstbeschreibung eines professionellen Radteams $\quad 66$

5. Das Team Blau - Analyse der im Team Blau geführten Interviews $\quad 69$

$5.1 \quad$ Herr Klar - Der Kulturträger $\quad 69$

5.2 Erste Zusammenführung: Die institutionalisierte , sowohl als auch Kultur"

$\begin{array}{lll}5.3 & \text { Herr Mutz - Der Absteiger } & 87\end{array}$

5.3.1 Kommunikative Pathologien durch differenzierte Wahrnehmungen (1) Herr Mutz und Herr Klar - „Existenzieller Kampf oder positiver Druck“

5.4 Herr Sonne - Der Aufsteiger $\quad 96$

5.4.1 Kommunikative Pathologien durch differenzierte Wahrnehmungen (2) Herr Sonne und Herr Klar - „Es gibt keine Trainingspläne versus natürlich gibt es die“ $\quad 101$

5.5 Herr Lefair - Der professionelle Enthusiast

5.5.1 Kommunikative Pathologien durch differenzierte Wahrnehmungen (3) Herr Lefair und Herr Klar - „Angriffslust oder sympathisches Hinterherfahren “ $\quad 106$

5.6 Herr Fern - Der Fremde

5.6.1 Kommunikative Pathologien durch differenzierte Wahrnehmungen (4) Herr Fern und Herr Klar - ,Kapitalgesellschaft versus emotionale Gemeinschaf “

5.7 Zweite Zusammenführung:

Die psychischen, sozialen und organisatorischen Faktoren im Team Blau

6. Fazit, Kritik und Ausblick

Nachwort von Wolfgang Weigand 
Für Judith 


\section{Vorwort}

Wird in der Presse dieser Tage das Thema Doping erwähnt, ließt man in der Regel über besser werdende Testverfahren, dichtere Kontrollen und härtere Strafen im Kampf gegen Dopingsünder. Die öffentliche Debatte um deviantes Verhalten im Sport verengt sich dabei auf medizinische und juristische Instrumente der Problemlösung zwischen denen straffällige und tatwillige Sportler eingekeilt werden sollen. Ziel ist, den Athleten von einer Manipulation abzuschrecken und einen sauberen Sport zu schaffen.

Nun weiß man nicht erst seit der Debatte um zunehmende Jugendgewalt, dass härtere Strafen nicht zwangsläufig zu weniger Gewaltdelikten führen - im Gegenteil. Vielmehr ist es wichtig, das soziale und gesellschaftliche Umfeld der Delinquenten in den Lösungsprozess einzubeziehen. Das gilt auch für den Sport. Der dopende Sportler ist kein devianter Einzeltäter, sondern ein Akteur im Schnittpunkt hochkomplexer Netzwerke, die sein Handeln ermöglichen und kanalisieren. Eine solche Perspektive wurde im Antidopingkampf bisher wenig berücksichtigt und ist unbequem. Sie bezieht uns alle in die Analyse der Ursachen des Dopings mit ein - als Eltern, Trainer, Politiker, Wirtschaftsakteure, Medienvertreter und Publikum.

Das soziale Umfeld des dopenden Sportlers gleicht zunächst dem des Hochleistungssportlers im Allgemeinen. Erst spezifische persönliche, gruppendynamische und organisatorische Zwänge trennen im weiteren Verlauf die Karrieren des Dopenden vom Nichtdopenden. So sind es auf persönlicher Ebene, durch die stetige Ausdehnung einer durchschnittlichen Karriere hervorgerufene, finanzielle Existenzängste die deviantes Handeln begünstigen. Die Soziologen Bette und Schimank bezeichnen diese Hochkostensituation, in der jede weitere Investition in den Sport den Erfolgsdruck potenziert, bei gleichzeitiger absoluter Minimierung anderer sozialer Aktivitäten, als „biographische Falle“. Ein Phänomen dem man durch parallel zur Sportkarriere laufende Ausbildungs- und Studienprogramme leicht entgegenwirken könnte. Auf gruppendynamischer Ebene findet sich das Problem der fehlenden Transparenz im Sinne eines: „Ich weiß nicht was der andere tut, also tue ich es vorsorglich auch.". Dabei führt die eigentümliche Verquickung von Einzelund Mannschaftssport im Profiradsport zu fragilen Gruppenkonstellationen in denen zwingender Egoismus durch monetäre Leistungen in Gruppendisziplin integriert werden muss. Mythen über mögliche Dopingmittel, die selten auf faktischem Wissen beruhen, bestimmen die Diskussionen zwischen Sportlern und Betreuern. Im Resultat wird keinem Sieger ein sauberer Sieg zugetraut und das gegenseitige Misstrauen im „Rüstungswettlauf“ ins Unermessliche getrieben. Aufklärung, Wertevermittlung und 
das Sichtbarmachen dieser Grauzone, wie es sich einige Pilotprojekte zum Ziel setzten, sind hier die richtigen Ansätze. Auf organisatorischer Ebene sind es mangelnde Vereinsführung, überfüllte den Trainingsplan diktierende Wettkampfkalender sowie überhöhte Leistungsvorgaben des Deutschen Olympischen Sportbundes, der im Zuge allgemeiner Kosteneinsparungen Olympiamedaillen fördert gute Leistungen, also Ergebnisse ab Platz 3, jedoch finanziell beschneidet, die Doping eher fördern als bekämpfen.

Auch aus systemischer Perspektive ist Doping für unsere Leistungsgesellschaft, der Sport als letztes faires Heldenepos dient, hochproblematisch. Sport erhält seinen Reiz und Sinn durch Regeln, Chancengleichheit, Grenzüberschreitung, fehlendes Stellvertreterhandeln und einen offenen Ausgang. Doping gefährdet diese Logik des Sports, in deren Lichte sich auch Akteure anderer gesellschaftlicher Felder gerne sonnen. Ein siegender Sportler ist dabei von besonderem Interesse und lässt das allgemeine sportliche Fairplay erst zum modernen Heldenepos werden. Olympische Medaillen spielen in der internationalen Politik ebenso eine Rolle wie im regionalen Wahlkampf. Gerne lassen sich politische Akteure mit Blumen und Pokalen ablichten. Dass Hochleistungssport in Verbindung mit Siegen für die Wirtschaft als Werbe- und Kommunikationsmittel hocheffizient ist, ist kein Geheimnis. Bleiben die Medien, die gerne über Helden berichten - oder Doping. Nur als Kuriosum erwecken Durchschnittsleistungen die mediale Aufmerksamkeit. Und wer verlangt nach den Ausnahmeathleten? Wir als Publikum und Katalysator dieses sich selbstbeschleunigenden Gefüges. So verstärken die externen Faktoren nur den Druck der Systemlogik, den sich der Sport mit dem Siegescode selbst setzt. Siege werden zum einzigen Trumpf und deren Ausnahme zur geforderten Alltäglichkeit.

Leise wird die letzte „Insel des Fairplay“ einer sonst entfesselten Leistungsgesellschaft von den Imperativen des Wirtschaftsystems besetzt. Wer glaubt, dass im Milliardengeschäft Sport mit bloßem Wasser gehandelt wird und werden kann, verschanzt sich hinter naiver Gläubigkeit. Erst wenn alle Beteiligten dieser „Konstellation“ (vgl. Bette; Schimank: 2006) ihre wechselseitige Mittäterschaft erkennen und sich am Präventionskampf beteiligen, ist ein nachhaltiger Erfolg denkbar. Einen gänzlich sauberen Sport gab es nie. Es wird ihn auch nie geben. Diese Einsicht mag traurig stimmen, sie wäre aber der Anfang einer ehrlich gefuhrten Auseinandersetzung die nicht des Dopings Willen, sondern um dessen Lösung geführt wird.

Ich hoffe, mit diesem Buch den beginnenden Paradigmenwechsel im Antidopingkampf zu unterstützen und um weitere Perspektiven bereichern zu können. 
Ich danke Judith für die vielen Gespräche, ihre unermüdliche Unterstützung und die Möglichkeit der Horizonterweiterung. Meinen Eltern Ingeborg und Werner Jahn danke ich für ihr Vertrauen. Professor Wolfgang Weigand bestärkte mich immer wieder im unkonventionellen Denken. Professor Stölting, Professor Hermanns und die Universität Potsdam stellten mir die „Insel der Wissenschaft“ im Meer des praktischen Vollzugs. Stefan Trommer bot mir freundschaftlichen Ausgleich zum theoretischen Dschungel. Schließlich danke ich Alexander Labrentz und Jana Mähne für ihre unverzichtbare redaktionelle Unterstützung.

Berlin im Mai 2008

Ronny Jahn 